\title{
Excess dose-related parameters (Vex, Rex, and iRex): novel predictors and late toxicity correlations in cervical cancer image-guided adaptive brachytherapy
}

\author{
Tissana Prasartseree, MD', Pittaya Dankulchai, MD!, Peter J. Hoskin, MD2.3 \\ 'Division of Radiation Oncology, Department of Radiology, Faculty of Medicine, Siriraj Hospital, Mahidol University, Bangkok, Thailand, \\ ${ }^{2}$ Mount Vernon Cancer Centre, Northwood, United Kingdom, ${ }^{3}$ University of Manchester, Manchester, United Kingdom
}

\begin{abstract}
Purpose: In this paper, excess dose is originally proposed to represent the dose outside the target volume that encompass only organs at risk (OARs), not the whole dose volume of isodose surface volume (ISV). By means of spatial consideration, excess dose-related parameters would also compensate inconsistent applicator positions and OARs motion, which may deviate the identical dose small-volume assumption of $\mathrm{D}_{2 \mathrm{cc}}$. Late toxicity correlations of these parameters were investigated.

Material and methods: A retrospective review was performed on cervical cancer high-dose-rate image-guided adaptive brachytherapy (HDR-IGABT). From ISVs of 60 to $100 \mathrm{~Gy} \mathrm{EQD}_{2}(\mathrm{a} / \beta=3)$, excess dose-related parameters were derived as following: toxicity negligible volume $\left(\mathrm{Vneg}=\mathrm{V}_{60}\right.$ of toxicity negligible organs; high-risk clinical target volume - HR-CTV, uterus, and vagina), excess dose volume (Vex = ISV - Vneg), Vneg normalized parameters of excess dose volume ratio $(\operatorname{Rex}=\mathrm{Vex} / \mathrm{Vneg})$, and indirect excess dose volume ratio (iRex $=\mathrm{ISV} / \mathrm{Vneg})$. Relationships between toxicity and these parameters were analyzed using a mean difference and a probit analysis method. Net reclassification indices (NRIs) were used to compare iRex60 and $\mathrm{D}_{2 \mathrm{cc}}$ gastrointestinal (GI) toxicity prediction.

Results: From 143 cases with an incidence of $34.9 \%$ and $10.5 \%$ of 3 -year grade $2-4$ GI and genitourinary (GU) toxicity, respectively, comparisons of means showed significant difference between grade 0-1 and 2-4 toxicities for late GI toxicity for all parameters, except ISV. There was a dose-response relationship with toxicity for each parameter across the range of 60-100 Gy EQD . ED $_{10}$ of iRex60 and iRex70 were 2.1 and 1.2, respectively. By comparing iRex60 and $\mathrm{D}_{2 c c^{\prime}}$ additive and absolute NRIs were +6.45 and $+7.69 \%$, respectively. The reclassification significantly occurred in range of 65-75 Gy of rectum $\mathrm{D}_{2 \mathrm{cc}}$.

Conclusions: Excess dose-related parameters, including Vex, Rex, and iRex, showed significant mean differences and parameter-toxicity relationships for late GI but not for GU toxicities. Positive NRIs suggest iRex60 utilization for spatial control of dose expansion, in addition to high-dose control with OAR small volumes. Further investigations are needed to define the optimum use of these predictors.
\end{abstract}

Key words: cervix cancer, excess dose-related parameters, indirect excess dose volume ratio (iRex).

\section{Purpose}

In image-guided adaptive brachytherapy (IGABT) for cervical cancer, inconsistent applicator positions, and movement of organs at risk (OARs) cause both inter- and intrafraction variation in dose conformation between each brachytherapy (BT) fraction $[1,2,3,4,5,6,7,8]$, which may affect toxicity prediction. The International Commission on Radiation Units and Measurements (ICRU) 89 mentioned the potential dose volume parameter called "isodose surface volume (ISV)" as the volume encompassed by clinically relevant equi-effective dose $\left(\mathrm{EQD}_{2}\right)$ levels of combined external beam radiation therapy
(EBRT) and BT doses [9]. The ISV includes not only the radiation target but also the mobile OARs, thus raising the probability of predicting OAR toxicity. In a retrospective series of 642 cervical cancer cases, a dose volume defined by the half product of height, width, and thickness (HWT), demonstrated significant correlations with rectal and soft tissue complications [10]. Total reference air kerma (TRAK), on which ISVs are based, was shown to correlate with morbidity $[11,12]$. For ISV, a large retrospective study of 1,253 patients failed to demonstrate any correlation between 60 and 160 Gy ISVs and tumor control [13]. The relationship between ISV and toxicity has not been studied. 
In the present study, the outside target volume dose or excess dose are originally proposed as the novel toxicity predictor. Since the excess dose includes only the space in which OAR may be located and moved, contrary to ISVs, of which the volume covers both target and OARs. Excess dose-related parameters are proposed to be more specific to OAR toxicity than ISV. Furthermore, spatial consideration of excess dose concept may compensate for the uncertainty of identical small-volume assumptions of dose-volume histogram parameters, e.g. $\mathrm{D}_{2 \mathrm{cc}}$ and $\mathrm{D}_{0.1 \mathrm{cc} \prime}$ from inconsistent applicator and OAR alignment.

In this study, excess dose volume (Vex) and its derivatives, including excess dose volume ratio (Rex) and indirect excess dose volume ratio (iRex) were proposed. Correlations between late gastrointestinal (GI) and genitourinary (GU) toxicity and these excess dose-related parameters were investigated as late toxicity predictors.

\section{Material and methods}

The study was approved by the Institutional Review Board, protocol number 713/2560 (EC1).

\section{Patient selection, treatment procedure, and clinical evaluation}

A retrospective review was performed on all cervical cancer patients receiving radical three-dimensional conformal radiotherapy (3D-CRT) with or without chemotherapy (CMT) between January 2012 and December 2015. Treatment was delivered in accordance with the Gynaecologic Groupe Européen de Curiethérapie-European Society for Radiation Oncology (GEC-ESTRO) recommendations $[14,15]$, using high-dose-rate image-guided adaptive brachytherapy (HDR-IGABT). In cases of central shielding, parametrial or pelvic lymph node boosts, or re-irradiation, patients were excluded due to interference with ISV dose calculation as well as those patients with a follow-up time less than 6 months or incomplete data.

IGABT was guided with computer tomography (CT) or magnetic resonance imaging (MRI). In every BT fraction, after catheterization, the bladder was filled with $50 \mathrm{ml}$ of contrast solution and rectal deflation using a rectal catheter was performed, while applicators were inserted and removed after radiation delivery.

Patient characteristics, imaging modalities, intracavitary (IC) with or without interstitial (IS) applicators, and dose parameters, including maximum dose to $2 \mathrm{cc}$ of OARs $\left(D_{2 c c}\right)$ were recorded. Late GI and GU toxicities were graded according to the Common Terminology Criteria for Adverse Events (CTCAE) version 5.0 [16]. Patients were classified into 0-1 and 2-4 grades of GI and GU late toxicities occurring six months or more after completion of the treatment. Lower GI toxicity included hemorrhage, proctitis, colitis, GI tract fistula, and perforation, and GU toxicities consisted of hematuria, cystitis, and GU fistula.

\section{Excess dose volume (Vex) and toxicity negligible volume (Vneg)}

Excess dose volume (Vex = ISV - Vneg) was defined as ISV with the toxicity negligible volume (Vneg) subtraction.
Vneg describes the radiation target and the close-to-applicator normal organs, which have less impact on quality of life. By hindering the applicator, Vneg was also considered as an OAR motion limiter, since it is located close to the applicators and reflects blockage of OARs motion toward the radiation source, sparing OARs irradiation.

\section{Excess dose volume ratio (Rex) and indirect excess dose volume ratio ( $i$ Rex)}

Absolute values of ISV and Vex would not reflect identical free space in each individual patient. Even with equal absolute values, ISVs and Vexs differ between each individual, due to different dose distributions and variation in the size of individual tumors and pelvic organs, which constitute Vneg. The individually varied conformations of ISV or Vex demonstrate unevenly covered OARs, leading to unequal toxicity correlations as described in Supplementary material. Hence, ISVs and Vexs would not alone be accurate, unless compared to Vneg. Excess dose volume ratio (Rex) and indirect excess dose volume ratio (iRex) were therefore proposed as the parameters, which should be normalized with Vneg and would compensate for conformationally omitted absolute values of ISV and Vex.

Rex, defined as Vex/Vneg = (ISV - Vneg $) /$ Vneg, normalizes values for Vex between each individual. Vex and Rex have the advantage of directly reflecting the permitted space for OAR motion, but subtracted volumes and ratios of Vex and Rex were not yet supported by the automatic optimization with current treatment planning systems (TPS). iRex, derived from ISV/Vneg, was an alternative parameter of Rex, and was obtainable from automatic optimization in current TPSs. iRex was therefore favored as a potential excess dose-related parameter.

\section{Proposed parameter delineation and calculation in each BT fraction}

Each of the novel parameters were generated as follows: ISVs were transformed from isodose lines of absorbed doses corresponding to total $\mathrm{EQD}_{2}(\mathrm{a} / \beta=3)$ of $60,70,80$, 90, and 100 Gy from the combined EBRT and BT dose, for example, absorbed dose of 2.34 Gy for one fraction corresponded to a total $\mathrm{EQD}_{2}(\mathrm{a} / \beta=3)$ of $60 \mathrm{~Gy}$ (Supplementary material). Vneg was constituted from high-risk clinical target volume (HR-CTV) and the toxicity negligible organs, such as uterus and vagina. Uterine and vaginal toxicities were considered negligible in this study, compared to bowel and bladder toxicities. Delineation of toxicity negligible organs included HR-CTV, uterus up to fundus, and vagina downward below lower border of generated ISV60s. Vneg60 was defined as volume of Vneg exposed to $60 \mathrm{~Gy} \mathrm{EQD}_{2}(\mathrm{a} / \beta=3)$ and was used in Vex delineation as well as in Rex and iRex calculations.

Vex (= ISV60, .., 100 - Vneg60) was generated by subtracting Vneg60 from each corresponding dose of ISV.

Each Rex (= Vex60, ..., 100/Vneg60) was calculated by dividing corresponding Vex with Vneg60, and similarly, ISV was divided by Vneg60 for iRex (= ISV60, ..., 100/Vneg60).

Delineations and ratio calculations are summarized in Figure 1. 
A Isodose surface volume (ISV) $60-100 \mathrm{~Gy} \mathrm{EQD}_{2}(\alpha / \beta=3)$
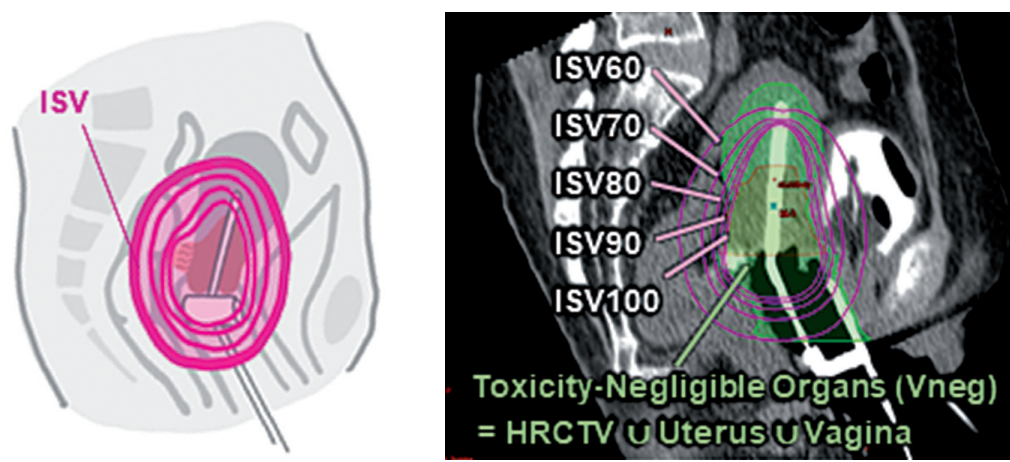

B

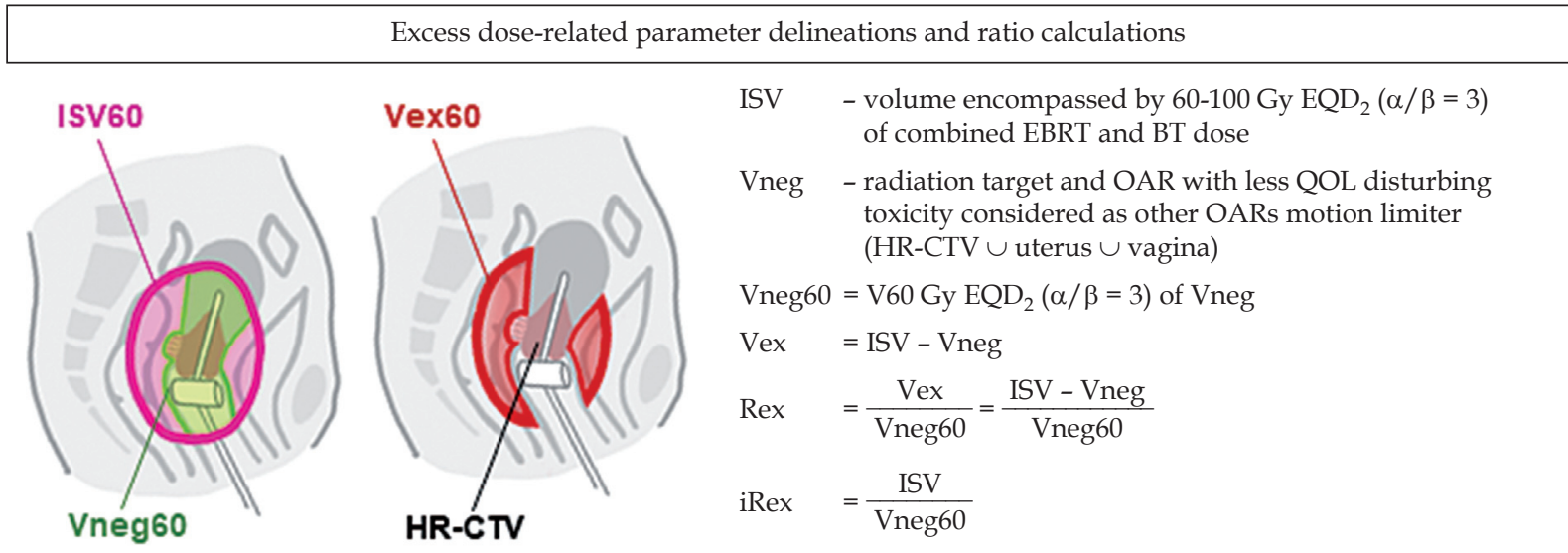

C

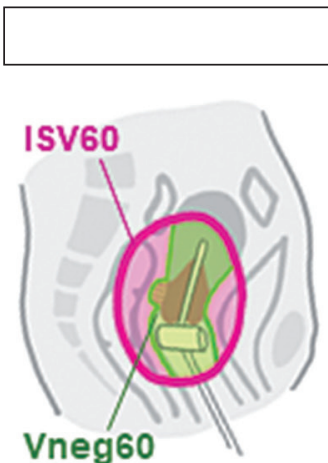

ISV60

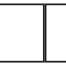

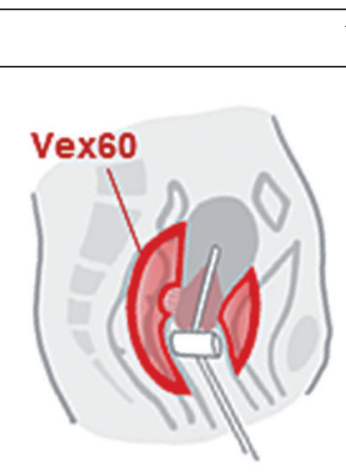

Vex60

D
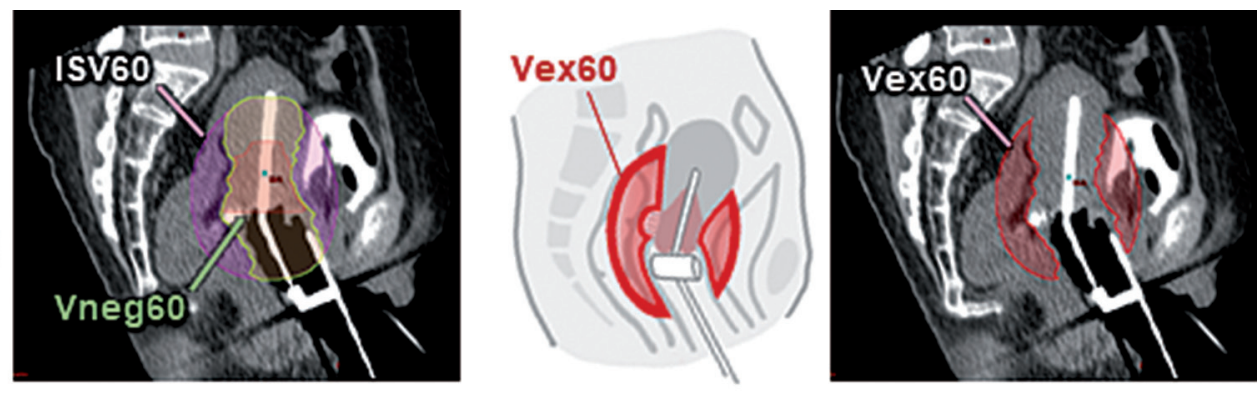

ISV70

Vex70
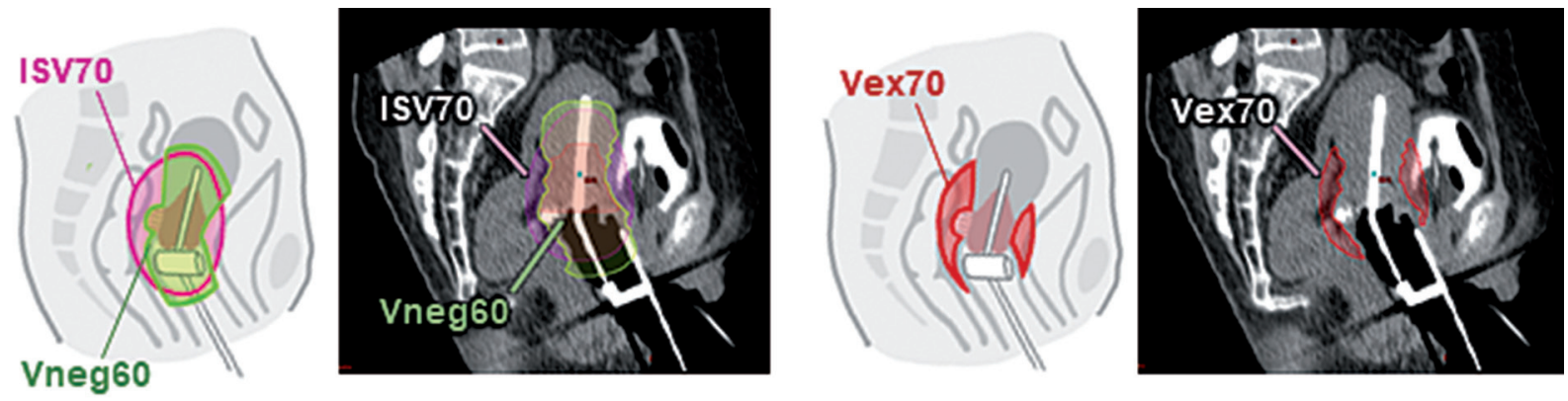

Fig. 1. A) Isodose surface volume (ISV) encompassing organs at risk, delineated in pink lines. Toxicity negligible organs included HR-CTV, uterus, and vagina delineated in the green line. B) Excess dose-related parameter delineations and ratio calculations. C) Depicts ISV60 and Vex60 example. D) Depicts ISV70 and Vex70 example

$V$ Veg - toxicity negligible volume, Vex-excess dose volume, Rex-excess dose volume ratio, iRex - indirect excess dose volume ratio, ISV60 - isodose surface volume of $60 \mathrm{~Gy} E Q D_{2}$, Vex60 - excess dose volume of $60 \mathrm{~Gy} E Q D_{2}, Q O L$ - quality of life 


\section{Toxicity - novel parameter correlation analysis and statistical method}

Mean ISV, Vex, Rex, and iRex data for every patient were calculated for each of the prescribed 3-5 fractions. Relationships between toxicity and novel parameters were analyzed for mean difference, and probit analysis was performed using PASW statistics, version 18.0.0. Mathematical means of these mean values were compared between grade 0-1 and 2-4 toxicities, using MannWhitney test. The dose-response relationship between means of parameters and grade 2-4 toxicities was analyzed with probit analysis.

\section{iRex60 vs. $D_{2 c c}$ GI toxicity prediction reclassification analysis}

Net reclassification indices (NRI) [17] were used to compare iRex60 and $\mathrm{D}_{2 \mathrm{cc}}$ GI toxicity prediction. An effective dose for $10 \%$ and $15 \%$ toxicity probability $\left(\mathrm{ED}_{10}\right.$ and $E D_{15}$ ) cut points of 2.1 and 2.3 from the iRex 60 probit result were used, while $\mathrm{D}_{2 \mathrm{cc}}$ values of 65 and $75 \mathrm{~Gy}$ for rectum were selected as defined in the image-guided intensity-modulated external beam radiochemotherapy and MRI-based adaptive BRAchytherapy in locally advanced CErvical cancer (EMBRACE II) protocol $[18,19]$.

Table 1. Patient characteristics (median follow-up time, 37 months)

\begin{tabular}{|c|c|c|c|c|c|c|c|}
\hline \multirow[t]{2}{*}{ Parameters } & \multirow{2}{*}{$\begin{array}{l}\text { Total } \\
n(\%)\end{array}$} & \multicolumn{3}{|c|}{ GI toxicity } & \multicolumn{3}{|c|}{ GU toxicity } \\
\hline & & Grade $0-1$ & Grade $2-4$ & $p$-value & Grade $0-1$ & Grade $2-4$ & $p$-value \\
\hline Total number (\%) & 143 & $93(65.1 \%)$ & $50(34.9 \%)$ & & $128(89.5 \%)$ & $15(10.5 \%)$ & \\
\hline Age (years), median (range) & $57(24-84)$ & $53(24-84)$ & $62(24-84)$ & $<0.001^{t}$ & $58(32-84)$ & $47(24-79)$ & $0.019^{t}$ \\
\hline \multicolumn{8}{|l|}{ FIGO 2009 staging } \\
\hline IB1 & $4(2.8 \%)$ & 0 & 4 & $0.198 x$ & 3 & 1 & $0.383^{x}$ \\
\hline IB2 & $7(4.9 \%)$ & 6 & 1 & - & 7 & 0 & - \\
\hline$\| \mathrm{A}$ & 17 (11.9\%) & 10 & 7 & - & 17 & 0 & - \\
\hline IIB & $44(30.8 \%)$ & 29 & 15 & - & 36 & 8 & - \\
\hline IIIA & $2(1.4 \%)$ & 0 & 2 & - & 2 & 0 & - \\
\hline$\| I I B$ & $59(41.3 \%)$ & 39 & 20 & - & 54 & 5 & - \\
\hline IVA & $10(6.9 \%)$ & 9 & 1 & - & 9 & 1 & - \\
\hline \multicolumn{8}{|l|}{ Concurrent chemotherapy } \\
\hline Yes & $130(90.9 \%)$ & 84 & 46 & $0.739 x$ & 117 & 13 & $0.546 x$ \\
\hline No & $13(9.1 \%)$ & 9 & 4 & - & 11 & 2 & - \\
\hline \multicolumn{8}{|l|}{ Imaging } \\
\hline CT-based BT & $101(70.6 \%)$ & 65 & 36 & $0.792^{x}$ & 92 & 9 & $0.338 x$ \\
\hline MRI-based BT & $42(29.4 \%)$ & 28 & 14 & - & 36 & 6 & - \\
\hline \multicolumn{8}{|l|}{ Applicator } \\
\hline IC alone & $84(58.7 \%)$ & 52 & 32 & $0.349 x$ & 75 & 9 & $0.917 x$ \\
\hline IC/IS & $59(41.3 \%)$ & 41 & 18 & & 53 & 6 & - \\
\hline Mean HR-CTV $\pm S D, c c$ & $35.12 \pm 25.40$ & $36.37 \pm 28.88$ & $32.79 \pm 17.15$ & $0.423^{t}$ & $35.35 \pm 26.31$ & $33.07 \pm 16.01$ & $0.743^{t}$ \\
\hline \multicolumn{8}{|l|}{$\begin{array}{l}\text { Mean } D_{2 c c} \pm S D \text {, Gy } \\
E_{2}(a / \beta=3)\end{array}$} \\
\hline Rectum & $69.75 \pm 5.95$ & $68.55 \pm 5.48$ & $71.98 \pm 6.18$ & $0.001^{t}$ & - & - & - \\
\hline Sigmoid & $60.12 \pm 6.33$ & $60.17 \pm 6.37$ & $60.02 \pm 6.32$ & $0.887^{t}$ & - & - & - \\
\hline Bowel & $64.78 \pm 10.50$ & $63.86 \pm 10.24$ & $66.49 \pm 10.88$ & $0.154^{t}$ & - & - & - \\
\hline Bladder & $85.08 \pm 7.08$ & - & - & - & $84.96 \pm 7.25$ & $85.95 \pm 5.72$ & $0.591^{t}$ \\
\hline
\end{tabular}

$D_{2 c c}$ - maximum dose to $2 C C, E Q D_{2}-2$ Gy equi-effective dose, HR-CTV - clinical target volume for high-risk region, IC - intracavitary, IS - interstitial implantation, $n$-number, $S D$ - standard deviation, ${ }^{t}-$ Student's t-test, $x$ - chi-square test 


\section{Results}

A total of 143 cases from 364 cervical cancer patients treated over the study period were reviewed. 221 cases were excluded due to central shielding $(n=92)$, parametrial and pelvic lymph nodal boost $(n=104)$, pelvic reirradiation $(n=2)$, and follow-up time less than 6 months or incomplete data $(n=23)$. Median follow-up time was

A
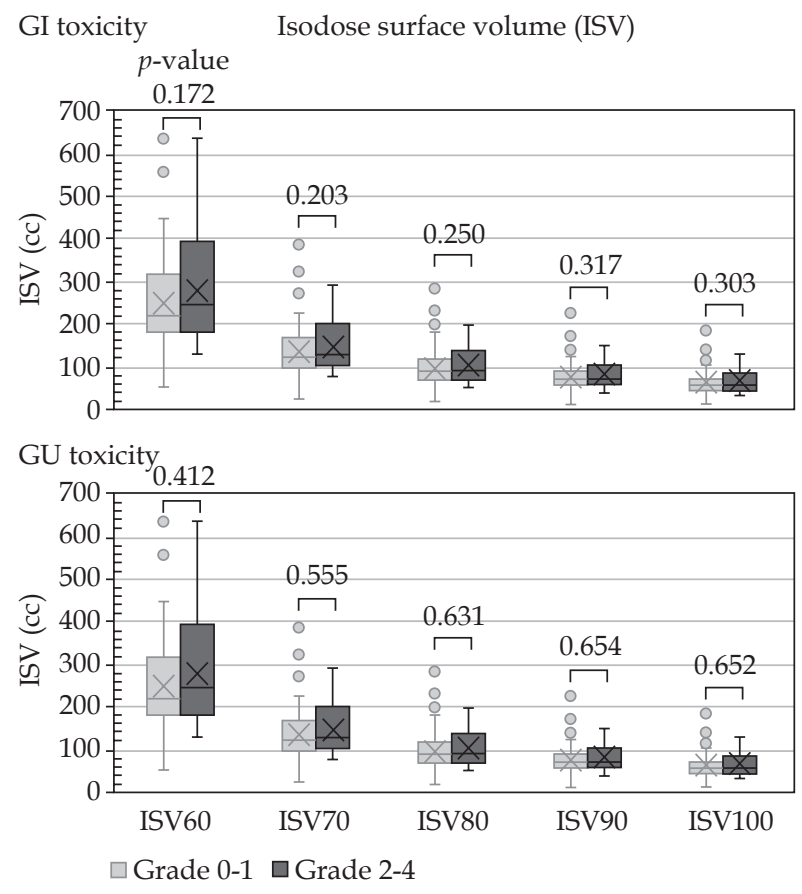

C

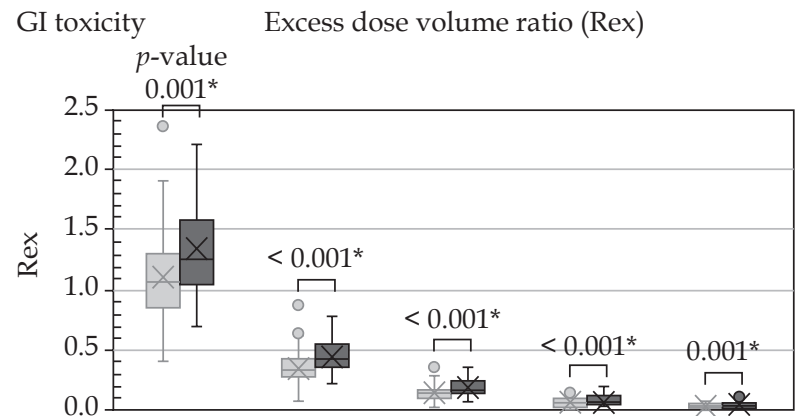

GU toxicity

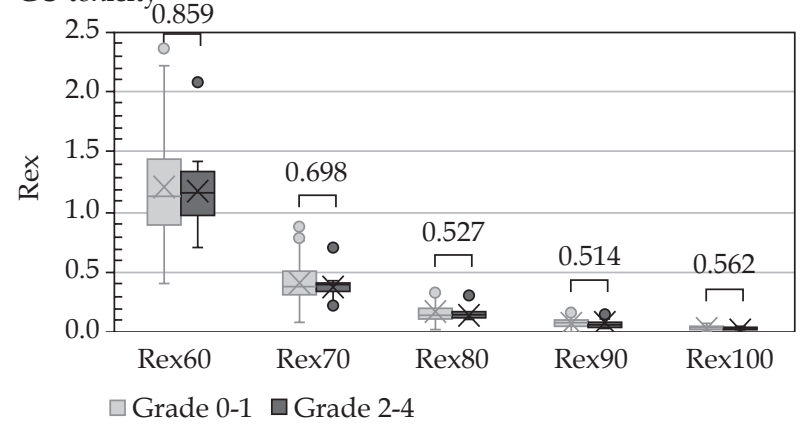

37 (range, 6-70) months. Patient characteristics and DVH parameters are presented in Table 1.

For BT, imaging modalities were classified into MRIbased and CT-based BT. MRI-based BT included patients who underwent MRI for every fraction (MR alone) or MRI for only first fraction of BT (MR first fraction), which accounted for $42(29.4 \%)$ patients. CT-based BT includ-

\section{B}

GI toxicity Excess dose volume (Vex)

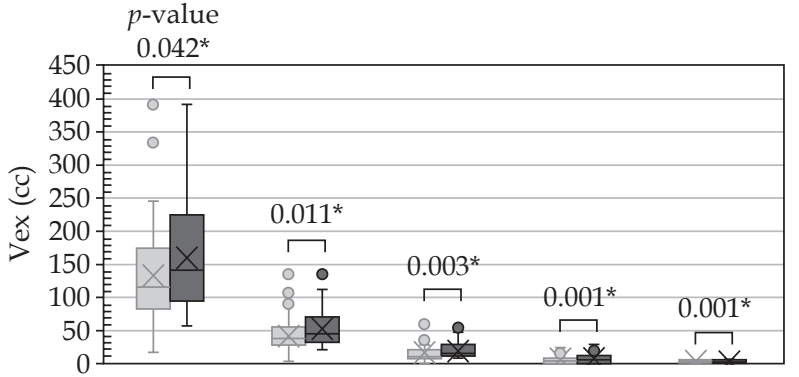

GU toxicity

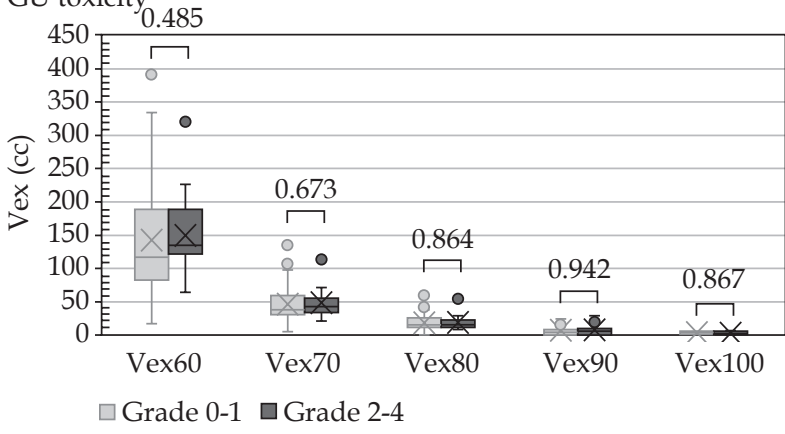

D

GI toxicity Indirect excess dose volume ratio (iRex)

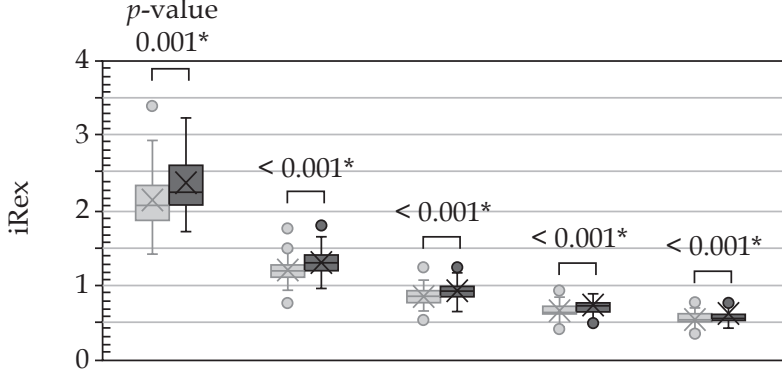

GU toxicity

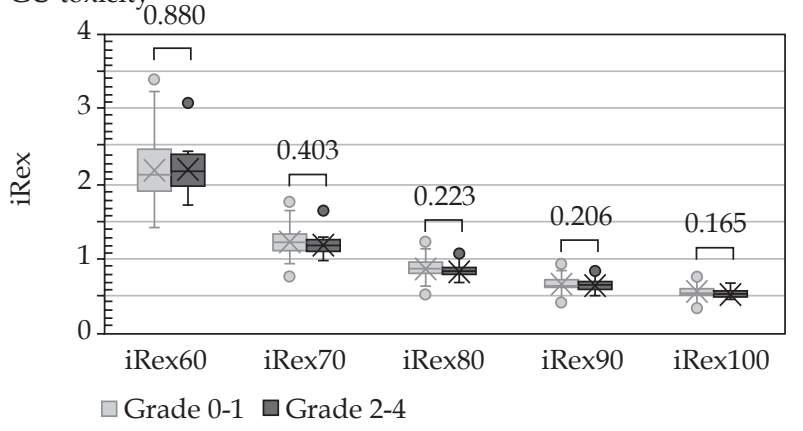

Fig. 2. Boxplots of mean of ISV, Vex, Rex, and iRex according to grade 0-1 and 2-4 GI and GU toxicities 
ed patients who underwent only CT for every fraction (CT alone) with or without fusion of pre-brachytherapy MRI (MR pre-BT), which occurred in 101 (70.6\%) patients. IC applicators were used for $84(58.7 \%)$ patients and in combination with IS for $59(41.3 \%)$ patients.

The total number of grade 2-4 late GI toxicities was 50 (34.9\%), while late GU toxicities was $15(10.5 \%)$. The total number of grade 3-4 late GI toxicities was 19 (13.3\%), while

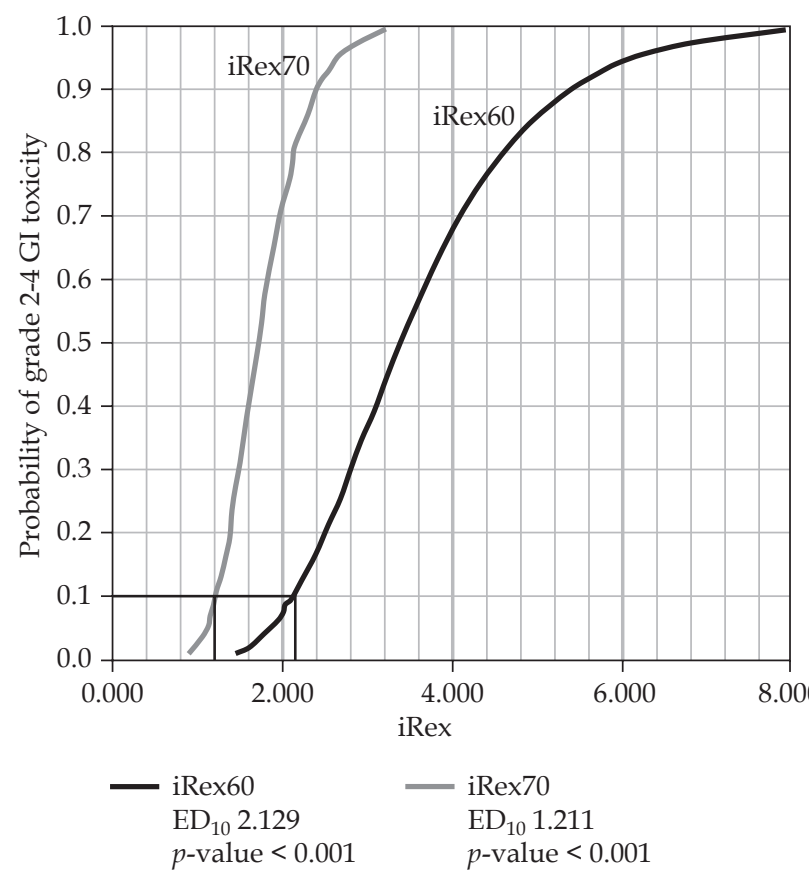

Fig. 3. Dose-response relationship of indirect excess dose volume ratio (iRex) and grade $2-4$ late GI toxicity by probit analysis

A No. of grade 2-4 GI late toxicities ( $n=50,34.9 \%$ of total)

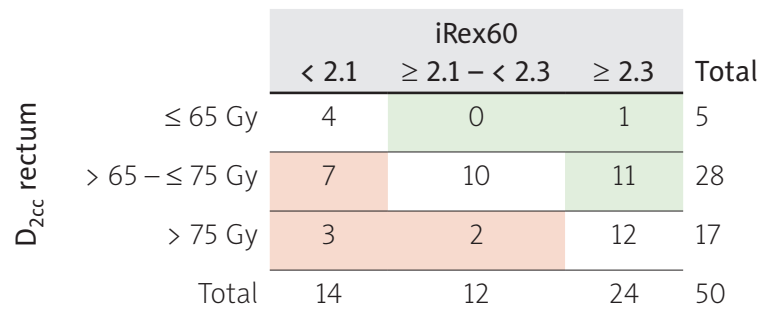

late GU toxicities was 5 (3.5\%). All 19 cases of grade 3 and 4 late GI toxicities were confirmed with endoscopic examination, and included 12 rectal, 3 rectosigmoid, 2 anorectal, and 1 case of both sigmoid and rectal telangiectasia. The remaining patient developed a grade 3 rectal ulcer with anal telangiectasia at 1 year after the treatment, followed by a grade 4 jejunal perforation, 5 years after the treatment.

The mathematical mean of mean Vex, Rex, and iRex for each patient demonstrated significant differences between grade $0-1$ and 2-4 late GI toxicity from 60 to 100 Gy $\mathrm{EQD}_{2}(\mathrm{a} / \beta=3)$. No significant mean differences were observed for ISV and all late GU toxicities (Figure 2 and Supplementary material).

There was a significant correlation with toxicity at each radiation dose for all parameters studied (see Supplementary material). The mean ISV exhibited a significant probit analysis for 70 to $100 \mathrm{~Gy} \mathrm{EQD}_{2}(\mathrm{a} / \beta=3)$ of ISV (ISV70 to ISV100), except for ISV60. The mean Vex showed a significant dose-response relationship with grade 2-4 late GI toxicities for Vex80 and Vex90. The mean Rex demonstrated a significant dose-response relationship with grade 2-4 late GI toxicities for Rex60. The mean iRex exhibited a significant dose-response relationship with grade 2-4 late GI toxicities for iRex60 and iRex70, with an $\mathrm{ED}_{10}$ of 2.129 and 1.211, respectively (Figure 3 and Supplementary material). When comparing iRex60 and $\mathrm{D}_{2 \mathrm{cc}}$ toxicity prediction, additive and absolute NRIs were +6.45 and $+7.69 \%$, respectively. The reclassification occurred most strongly in the 65-75 Gy range for the $D_{2 c c}$ of rectum (Figure 4).

\section{Discussion}

In this study, the discrepantly higher toxicity was observed, comparing to tolerable D2cc doses. As hypothesized, the effect of inconsistent applicator positions and

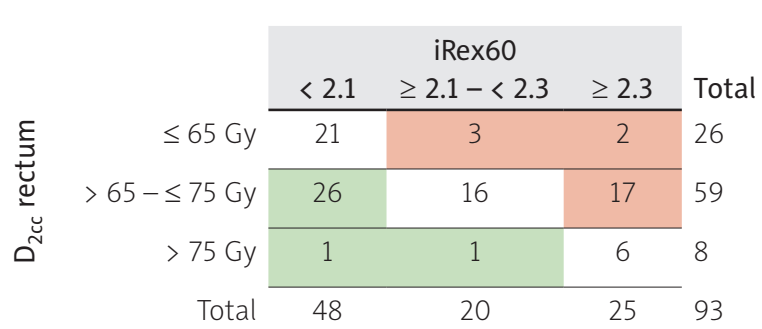

C Reclassification indices calculation

\begin{tabular}{|c|c|c|c|}
\hline & $\begin{array}{c}\text { Grade 2-4 } \\
\text { (events) }\end{array}$ & $\begin{array}{c}\text { Grade 0-1 } \\
\text { (non-events) }\end{array}$ & \\
\hline Correct reclassification & 12 & 28 & \multirow{3}{*}{$\begin{array}{ll}\text { Additive NRI } & +6.45 \\
\text { Absolute NRI } & +7.69 \% \\
\end{array}$} \\
\hline Incorrect reclassification & 12 & 22 & \\
\hline Net reclassification & 0 & 6 & \\
\hline
\end{tabular}

Fig. 4. iRex60 vs. $\mathrm{D}_{2 \mathrm{cc}}$ GI toxicity prediction reclassification analysis

$D_{2 c c}$ - maximum dose to $2 c c$, iRex60 - indirect excess dose volume ratio of $60 \mathrm{~Gy} E Q D_{2}(\alpha / \beta=3), N R I-$ net reclassification indices 
OARs motion may deviate the identical dose-volume assumption of small-volume dose. Excess dose-related parameters, proposed to represent the dose outside the target and to compensate the OAR motion factor, established significant correlations with late GI toxicity only for Vex, Rex, and iRex, but not ISV. No correlation was observed with GU toxicity.

There was no significant correlation between ISV and GI toxicity. In previous studies, which have reported significant correlation $[10,11,12]$, ISV or TRAK and toxicity correlation may have been confused by the dose outside target, not the whole dose volume.

Vex showed a significant relationship with GI toxicity, with a significant dose-response relation at 80-90 Gy. The lack of a dose-response relation for Vex100 resulted from the exceedingly small volumes of Vex100. The absence of toxicity correlation with Vex60 and Vex70 could reflect the uncertainty in toxicity prediction using absolute dose volumes at low doses. At low-dose levels, with larger territory of dose coverage, the distribution of dose is more varied compared to high-dose level. The equal absolute values for low-dose levels of ISV and Vex between each individual fraction could not reflect the different shape and coverage of the OAR outside Vneg. Therefore, the poor correlation with toxicity at low-dose levels was demonstrated by examples included in Supplementary material.

In contrast, for Rex and iRex, the lower dose levels were predictive of toxicity, in particular Rex60 and iRex60-70, comparable to the higher predictive dose levels of ISV and Vex. The low-dose prediction of Rex and iRex emphasizes the normalization effect on Vneg from calculating Rex and iRex, which compensates for the varied conformations of ISV and Vex. Both Rex and iRex demonstrated the same significant correlations between Rex60, iRex60, and iRex70 for late GI toxicity. These results confirm that iRex may be used instead of Rex.

The absence of a relation between GU toxicity and any parameter may be explained by the low number of events $(10.5 \%)$, compared to GI toxicity events (34.9\%).

Excess dose-related parameters could be more accurate as toxicity predictors because they compensate for OAR motion, which is not captured in dose-volume histogram $(\mathrm{DVH})$ parameters. Based on the analysis presented here, Rex and iRex were recommended as having a significant correlation with toxicity in low-dose regions and the Vneg relative properties. Positive NRIs also suggested iRex60 utilization for dose expansion spatial control, in addition to high-dose control with OAR small volumes.

Although, $\operatorname{Rex}(\operatorname{Rex}=\mathrm{Vex} / \mathrm{Vneg})$ directly reflects the excess dose, unfortunately this was not currently derived during the optimization process within a commercial TPS. However, iRex (iRex = ISV/Vneg) as an indirect measure of Rex, was produced by the TPS, and it was therefore proposed that iRex was used until Rex could be produced by the TPS automatically.

A limitation of these conclusions was that the comparison of ISV, Vex, Rex, and iRex with dose to small volumes $\left(D_{2 c c}\right.$ and $\left.D_{0.1 c c}\right)$ in predicting toxicity has not been fully validated. Toxicity measurement was retrospective with inherent inaccuracies. There was a variation in im- aging, using both CT and MRI, which would have altered OAR volume accuracy; CT volumes were tending to be larger than those defined by MRI. This study assumed a uniform dose of EBRT. Its validity when a pelvic boost was used, which would interfere with ISV dose calculation should yet be explored. Future studies with multivariate analysis as well as prospective studies and DVH parameter comparison are crucial in further validation and development of these novel toxicity predictors.

\section{Conclusions}

Excess dose-related parameters, including Vex, Rex, and iRex, were significantly related to late GI but not GU toxicity. iRex60 is the most suitable parameter, as it can be derived directly from a commercial TPS, with an $\mathrm{ED}_{10}$ of 2.1. Further investigations are needed for robust validation of these toxicity predictors to be used for spatial control of dose expansion in addition to control of high-dose regions by conventional DVH parameters for OAR small volumes, such as $\mathrm{D}_{2 \mathrm{cc}}$ and $\mathrm{D}_{0.1 \mathrm{cc}}$.

\section{Funding}

There were no specific sources of funding for this project. PJH is supported by the NIHR Manchester Biomedical Research Centre.

\section{Disclosure}

The authors report no conflict of interest.

\section{References}

1. Mazeron R, Champoudry J, Gilmore J et al. Intrafractional organs movement in three-dimensional image-guided adaptive pulsed-dose-rate cervical cancer brachytherapy: Assessment and dosimetric impact. Brachytherapy 2015; 14: 260-266.

2. Nesvacil N, Tanderup K, Hellebust TP et al. A multicentre comparison of the dosimetric impact of inter- and intrafractional anatomical variations in fractionated cervix cancer brachytherapy. Radiother Oncol 2013; 107: 20-25.

3. Morgia M, Cuartero J, Walsh L et al. Tumor and normal tissue dosimetry changes during MR-guided pulsed-doserate (PDR) brachytherapy for cervical cancer. Radiother Oncol 2013; 107: 46-51.

4. Hellebust TP, Dale E, Skjonsberg A et al. Inter fraction variations in rectum and bladder volumes and dose distributions during high dose rate brachytherapy treatment of the uterine cervix investigated by repetitive CT-examinations. Radiother Oncol 2001; 60: 273-280.

5. Jamema SV, Mahantshetty U, Tanderup K et al. Inter-application variation of dose and spatial location of $\mathrm{D}(2 \mathrm{~cm}(3))$ volumes of OARs during MR image based cervix brachytherapy. Radiother Oncol 2013; 107: 58-62.

6. Lang S, Nesvacil N, Kirisits C et al. Uncertainty analysis for 3D image-based cervix cancer brachytherapy by repetitive MR imaging: Assessment of DVH-variations between two HDR fractions within one applicator insertion and their clinical relevance. Radiother Oncol 2013; 107: 26-31.

7. Anderson C, Lowe G, Wills $R$ et al. Critical structure movement in cervix brachytherapy. Radiother Oncol 2013; 107: 39-45.

8. Simha V, Patel FD, Sharma SC et al. Evaluation of intrafraction motion of the organs at risk in image-based brachytherapy of cervical cancer. Brachytherapy 2014; 13: 562-567. 
9. International Commission on Radiation Units \& Measurements. Prescribing, recording, and reporting brachytherapy for cancer of the cervix (ICRU Report 89). Bethesda, 2013.

10. Barillot I, Horiot JC, Maingon P et al. Impact on treatment outcome and late effects of customized treatment planning in cervix carcinomas: baseline results to compare new strategies. Int J Radiat Oncol Biol Phys 2000; 48: 189-200.

11. Hunter R, Wilkinson J, Swindell R. 142 Oral Volume in intracavitary therapy; the first proof that it is clinically significant. Radiother Oncol 2002; 64: S47-48.

12. Bockel S, Escande A, Dumas I et al. Total reference air kerma is associated with late bowel morbidity in locally advanced cervical cancer patients treated with image-guided adaptive brachytherapy. J Clin Med 2019; 8: 125.

13. Grigsby PW, Williamson JF, Clifford Chao KS et al. Cervical tumor control evaluated with ICRU38 reference volume and integrated reference air kerma. Radiother Oncol 2000; 58: 19-23.

14. Haie-Meder C, Potter R, Van Limbergen E et al. Recommendations from Gynaecological (GYN) GEC-ESTRO Working Group (I): Concepts and terms in 3D image based 3D treatment planning in cervix cancer brachytherapy with emphasis on MRI assessment of GTV and CTV. Radiother Oncol 2005; 74: 235-245.

15. Pötter R, Haie-Meder C, Van Limbergen E et al. Recommendations from gynaecological (GYN) GEC ESTRO working group (II): Concepts and terms in 3D image-based treatment planning in cervix cancer brachytherapy-3D dose volume parameters and aspects of 3D image-based anatomy, radiation physics, radiobiology. Radiother Oncol 2006; 78: 67-77.

16. Common Terminology Criteria for Adverse Events (CTCAE) [Internet]. Cancer Therapy Evaluation Program (CTEP). Available from: https://ctep.cancer.gov/protocolDevelopment/electronic_applications/ctc.htm

17. Alba AC, Agoritsas T, Walsh M et al. Discrimination and calibration of clinical prediction models: users' guides to the medical literature. JAMA 2017; 318: 1377-1384.

18. Pötter R, Tanderup K, Kirisits C et al.; EMBRACE Collaborative Group. The EMBRACE II study: The outcome and prospect of two decades of evolution within the GEC-ESTRO GYN working group and the EMBRACE studies. Clin Transl Radiat Oncol 2018; 9: 48-60.

19. Tanderup K, Pötter R, Lindegaard J et al. Image guided intensity modulated External beam radiochemotherapy and MRI based adaptive BRAchytherapy in locally advanced CErvical cancer (EMBRACE-II) study protocol v.1.0 [Internet]. Available from: https://www.embracestudy.dk/UserUpload/ PublicDocuments/EMBRACE\%20II\%20Protocol.pdf 


\section{Supplementary material}

Table S1. Fractional absorbed doses corresponded to equi-effective dose $\left(E Q D_{2}\right)(\alpha / \beta=3)$ of $60,70,80,90$, and 100 Gy from combined external beam radiation therapy (EBRT) and brachytherapy (BT) dose

\begin{tabular}{lcccccc} 
Total EBRT & Number of BT & \multicolumn{5}{c}{ Fractional absorbed doses corresponded to $\mathrm{EQD}_{2}(\alpha / \beta=3)$, Gy } \\
\cline { 3 - 7 } dose, Gy & fractions & ISV60 & ISV70 & ISV80 & ISV90 & ISV100 \\
\hline 46 & 4 & 2.94 & 4.18 & 5.19 & 6.07 & 6.85 \\
\hline 46 & 5 & 2.53 & 3.62 & 4.52 & 5.30 & 6.00 \\
\hline 50 & 3 & 2.85 & 4.47 & 5.73 & 6.80 & 7.75 \\
\hline 50 & 4 & 2.34 & 3.72 & 4.80 & 5.73 & 6.55 \\
\hline 50 & 5 & 2.00 & 3.22 & 4.18 & 5.00 & 5.73
\end{tabular}

Table S2. The comparison of mean differences of all parameters between grade 0-1 and 2-4 late toxicity groups, with median follow-up time of 37 months

\begin{tabular}{|c|c|c|c|c|c|c|c|c|}
\hline \multirow[t]{2}{*}{ Grade } & & & \multicolumn{3}{|c|}{ GI toxicity } & \multicolumn{3}{|c|}{ GU toxicity } \\
\hline & & & Grade $0-1$ & Grade 2-4 & $p$-value & Grade $0-1$ & Grade $2-4$ & $p$-value \\
\hline \multirow{5}{*}{$\begin{array}{l}\text { Isodose surface } \\
\text { volume } \\
\text { (ISV) } \\
\text { Mean } \pm S D \text {, cc }\end{array}$} & ISV60 & $261.5 \pm 117.5$ & $251.2 \pm 113.0$ & $280.6 \pm 124.3$ & 0.172 & $260.2 \pm 119.8$ & $272.4 \pm 98.3$ & 0.412 \\
\hline & ISV70 & $143.3 \pm 59.7$ & $139.0 \pm 60.2$ & $151.4 \pm 58.7$ & 0.203 & $143.0 \pm 61.0$ & $146.5 \pm 48.8$ & 0.555 \\
\hline & ISV80 & $101.2 \pm 41.1$ & $98.4 \pm 41.9$ & $106.2 \pm 39.3$ & 0.250 & $101.0 \pm 42.1$ & $102.5 \pm 32.4$ & 0.631 \\
\hline & ISV90 & $78.9 \pm 31.7$ & $77.1 \pm 32.7$ & $82.2 \pm 29.7$ & 0.317 & $78.8 \pm 32.4$ & $79.9 \pm 24.8$ & 0.654 \\
\hline & ISV100 & $65.2 \pm 26.2$ & $63.7 \pm 27.0$ & $68.0 \pm 24.7$ & 0.303 & $65.1 \pm 26.9$ & $65.8 \pm 20.5$ & 0.652 \\
\hline \multirow{5}{*}{$\begin{array}{l}\text { Excess dose volume } \\
(\text { Vex }=\text { ISV - Vneg) } \\
\text { Mean } \pm S D, c C\end{array}$} & Vex60 & $142.7 \pm 75.8$ & $132.6 \pm 70.0$ & $161.5 \pm 83.1$ & 0.042 & $142.1 \pm 77.1$ & $148.0 \pm 66.0$ & 0.485 \\
\hline & Vex70 & $46.3 \pm 24.6$ & $42.3 \pm 22.6$ & $53.6 \pm 26.7$ & 0.011 & $46.3 \pm 25.0$ & $46.3 \pm 22.1$ & 0.673 \\
\hline & Vex80 & $18.1 \pm 10.7$ & $16.3 \pm 9.7$ & $21.6 \pm 11.7$ & 0.003 & $18.2 \pm 10.9$ & $17.8 \pm 9.5$ & 0.864 \\
\hline & Vex90 & $7.4 \pm 5.0$ & $6.4 \pm 4.5$ & $9.1 \pm 5.6$ & 0.001 & $7.4 \pm 5.1$ & $7.0 \pm 4.4$ & 0.942 \\
\hline & Vex100 & $3.1 \pm 2.6$ & $2.6 \pm 2.2$ & $4.0 \pm 2.9$ & 0.001 & $3.1 \pm 2.6$ & $2.9 \pm 2.3$ & 0.867 \\
\hline \multirow{5}{*}{$\begin{array}{l}\text { Excess dose volume } \\
\text { ratio } \\
(\text { Rex }=\text { Vex/Vneg) } \\
\text { Mean } \pm S D\end{array}$} & Rex60 & $1.20 \pm 0.40$ & $1.12 \pm 0.37$ & $1.36 \pm 0.42$ & 0.001 & $1.20 \pm 0.41$ & $1.19 \pm 0.33$ & 0.859 \\
\hline & Rex70 & $0.39 \pm 0.15$ & $0.36 \pm 0.13$ & $0.46 \pm 0.15$ & $<0.001$ & $0.40 \pm 0.15$ & $0.37 \pm 0.11$ & 0.698 \\
\hline & Rex80 & $0.16 \pm 0.07$ & $0.14 \pm 0.07$ & $0.19 \pm 0.07$ & $<0.001$ & $0.16 \pm 0.07$ & $0.14 \pm 0.05$ & 0.527 \\
\hline & Rex90 & $0.06 \pm 0.04$ & $0.06 \pm 0.04$ & $0.08 \pm 0.04$ & $<0.001$ & $0.07 \pm 0.04$ & $0.06 \pm 0.02$ & 0.514 \\
\hline & Rex100 & $0.03 \pm 0.02$ & $0.02 \pm 0.02$ & $0.04 \pm 0.02$ & 0.001 & $0.03 \pm 0.02$ & $0.02 \pm 0.01$ & 0.562 \\
\hline \multirow{5}{*}{$\begin{array}{l}\text { Indirect excess dose } \\
\text { volume ratio } \\
\text { (iRex }=\text { ISV/Vneg) } \\
\text { Mean } \pm \text { SD }\end{array}$} & iRex60 & $2.22 \pm 0.41$ & $2.13 \pm 0.37$ & $2.38 \pm 0.44$ & 0.001 & $2.22 \pm 0.42$ & $2.20 \pm 0.33$ & 0.880 \\
\hline & iRex70 & $1.22 \pm 0.17$ & $1.18 \pm 0.15$ & $1.30 \pm 0.18$ & $<0.001$ & $1.23 \pm 0.18$ & $1.19 \pm 0.15$ & 0.403 \\
\hline & iRex80 & $0.87 \pm 0.12$ & $0.84 \pm 0.11$ & $0.92 \pm 0.12$ & $<0.001$ & $0.87 \pm 0.12$ & $0.84 \pm 0.09$ & 0.223 \\
\hline & iRex90 & $0.68 \pm 0.09$ & $0.66 \pm 0.08$ & $0.71 \pm 0.08$ & $<0.001$ & $0.68 \pm 0.09$ & $0.65 \pm 0.07$ & 0.206 \\
\hline & iRex100 & $0.56 \pm 0.07$ & $0.54 \pm 0.07$ & $0.59 \pm 0.07$ & $<0.001$ & $0.56 \pm 0.07$ & $0.54 \pm 0.06$ & 0.165 \\
\hline
\end{tabular}


Table S3. Probability of isodose surface volume (ISV) for grade 2-4 late toxicities for the incidence rates shown in 95\% confidence interval

\begin{tabular}{lcccc} 
ISV & $5 \%$ & $10 \%$ & $20 \%$ & $p$-value \\
\hline GI toxicity & $134.9(128.8-140.7)$ & $194.6(189.0-200.0)$ & $303.2(296.6-310.2)$ & 0.078 \\
\hline ISV60 & $78.0(74.2-81.6)$ & $109.3(105.9-112.6)$ & $164.6(160.8-168.7)$ & 0.006 \\
\hline ISV70 & $56.0(53.2-58.6)$ & $77.6(75.2-80.0)$ & $115.4(112.6-118.2)$ & $<0.001$ \\
\hline ISV80 & $43.8(41.5-45.9)$ & $60.4(58.4-62.3)$ & $89.3(87.1-91.5)$ & $<0.001$ \\
\hline ISV90 & $36.3(34.5-38.0)$ & $50.1(48.4-51.6)$ & $73.9(72.1-75.7)$ & $<0.001$ \\
\hline ISV100 & & & $750.3(686.7-833.7)$ & 1.000 \\
\hline GU toxicity & $280.3(269.7-291.0)$ & $437.5(416.0-463.5)$ & $387.2(355.5-428.7)$ & 1.000 \\
\hline ISV60 & $150.2(144.8-155.7)$ & $230.5(219.7-243.5)$ & $269.5(247.3-298.6)$ & 1.000 \\
\hline ISV70 & $104.9(101.1-108.6)$ & $160.6(153.2-169.7)$ & $208.7(191.5-231.2)$ & 1.000 \\
\hline ISV80 & $81.5(78.6-84.5)$ & $124.7(118.10-131.7)$ & $171.3(157.4-189.5)$ & 1.000
\end{tabular}

Table S4. Probability of excess dose volume (Vex) for grade 2-4 late toxicities for the incidence rates shown in $95 \%$ confidence interval

\begin{tabular}{lcccc} 
Vex & $5 \%$ & $10 \%$ & $20 \%$ & $p$-value \\
\hline Gl toxicity & $70.8(67.5-74.1)$ & $106.1(102.8-109.3)$ & $173.0(168.7-177.5)$ & 0.851 \\
\hline Vex60 & $24.6(23.6-25.7)$ & $36.1(35.1-37.1)$ & $57.4(56.0-58.9)$ & 0.101 \\
\hline Vex70 & $9.54(9.03-10.02)$ & $14.2(13.7-14.7)$ & $23.1(22.4-23.9)$ & 0.001 \\
\hline Vex80 & $3.67(3.45-3.88)$ & $5.69(5.45-5.91)$ & $9.65(9.31-10.0)$ & $<0.001$ \\
\hline Vex90 & $1.32(1.24-1.39)$ & $2.21(2.13-2.30)$ & $4.15(4.01-4.30)$ & 0.273 \\
\hline Vex100 & & & $461.5(418.8-517.5)$ & 1.000 \\
\hline GU toxicity & $150.7(144.3-157.3)$ & $250.0(236.6-266.2)$ & $147.6(133.9-165.7)$ & 0.998 \\
\hline Vex60 & $47.3(45.2-49.3)$ & $79.1(74.9-84.2)$ & $63.8(57.2-72.6)$ & 0.317 \\
\hline Vex70 & $17.7(16.8-18.9)$ & $31.6(29.8-33.9)$ & $29.4(25.6-34.7)$ & 0.009 \\
\hline Vex80 & $6.90(6.46-7.34)$ & $13.3(12.3-14.5)$ & $15.8(13.6-18.8)$ & 0.768 \\
\hline Vex90 & $2.62(2.44-2.81)$ & $5.90(5.45-6.46)$ & & \\
\hline Vex100 & & & & \\
\hline
\end{tabular}


Table S5. Probability of excess dose volume ratio (Rex) for grade 2-4 late toxicities for the incidence rates shown in 95\% confidence interval

\begin{tabular}{llccc} 
Rex & \multicolumn{1}{c}{$5 \%$} & $10 \%$ & $20 \%$ & $p$-value \\
\hline GI toxicity & $0.884(0.856-0.909)$ & $1.105(1.081-1.128)$ & $1.449(1.422-1.477)$ & $<0.001$ \\
\hline Rex60 & $0.289(0.282-0.296)$ & $0.368(0.361-0.374)$ & $0.492(0.484-0.500)$ & 0.176 \\
\hline Rex70 & $0.104(0.101-0.107)$ & $0.140(0.137-0.143)$ & $0.201(0.197-0.205)$ & 0.722 \\
\hline Rex80 & $0.037(0.035-0.038)$ & $0.054(0.052-0.055)$ & $0.086(0.083-0.088)$ & 0.920 \\
\hline Rex90 & $0.012(0.011-0.013)$ & $0.020(0.019-0.021)$ & $0.037(0.036-0.038)$ & 1.000 \\
\hline Rex100 & $1.232(1.197-1.267)$ & $1.727(1.667-1.796)$ & $2.598(2.439-2.799)$ & 1.000 \\
\hline GU toxicity & $0.386(0.373-0.398)$ & $0.562(0.542-0.586)$ & $0.888(0.830-0.962)$ & 0.263 \\
\hline Rex60 & $0.147(0.141-0.154)$ & $0.231(0.220-0.244)$ & $0.399(0.366-0.442)$ & 0.019 \\
\hline Rex70 & $0.057(0.054-0.060)$ & $0.103(0.098-0.109)$ & $0.209(0.189-0.235)$ & 0.208 \\
\hline Rex80 & $0.023(0.021-0.024)$ & $0.050(0.046-0.054)$ & $0.129(0.112-0.152)$ & 0.998 \\
\hline Rex90 & & & & \\
\hline Rex100 & & & & \\
\hline
\end{tabular}

Table S6. Probability of indirect excess dose volume ratio (iRex) for grade 2-4 late toxicities for the incidence rates shown in $95 \%$ confidence interval

\begin{tabular}{llccc} 
iRex & \multicolumn{1}{c}{$5 \%$} & $10 \%$ & $20 \%$ & $p$-value \\
\hline GI toxicity & & & & \\
\hline iRex60 & $1.865(1.825-1.901)$ & $2.129(2.098-2.158)$ & $2.499(2.467-2.532)$ & $<0.001$ \\
\hline iRex70 & $1.099(1.085-1.112)$ & $1.211(1.201-1.222)$ & $1.363(1.352-1.375)$ & $<0.001$ \\
\hline iRex80 & $0.785(0.778-0.793)$ & $0.860(0.854-0.865)$ & $0.959(0.953-0.965)$ & 0.395 \\
\hline iRex90 & $0.615(0.610-0.621)$ & $0.670(0.666-0.674)$ & $0.743(0.739-0.748)$ & 1.000 \\
\hline iRex100 & $0.507(0.502-0.512)$ & $0.553(0.550-0.557)$ & $0.615(0.611-0.618)$ & 0.918 \\
\hline GU toxicity & & & $3.525(3.395-3.687)$ & 0.940 \\
\hline iRex60 & $2.265(2.226-2.302)$ & $2.766(2.709-2.833)$ & $1.772(1.714-1.846)$ & 0.681 \\
\hline iRex70 & $1.199(1.180-1.216)$ & $1.431(1.406-1.459)$ & $1.212(1.176-1.258)$ & 0.767 \\
\hline iRex80 & $0.841(0.829-0.853)$ & $0.992(0.977-1.010)$ & $0.926(0.900-0.958)$ & 0.986 \\
\hline iRex90 & $0.659(0.649-0.667)$ & $0.768(0.757-0.781)$ & $0.774(0.751-0.803)$ & 0.823 \\
\hline iRex100 & $0.541(0.533-0.548)$ & $0.636(0.627-0.647)$ & &
\end{tabular}



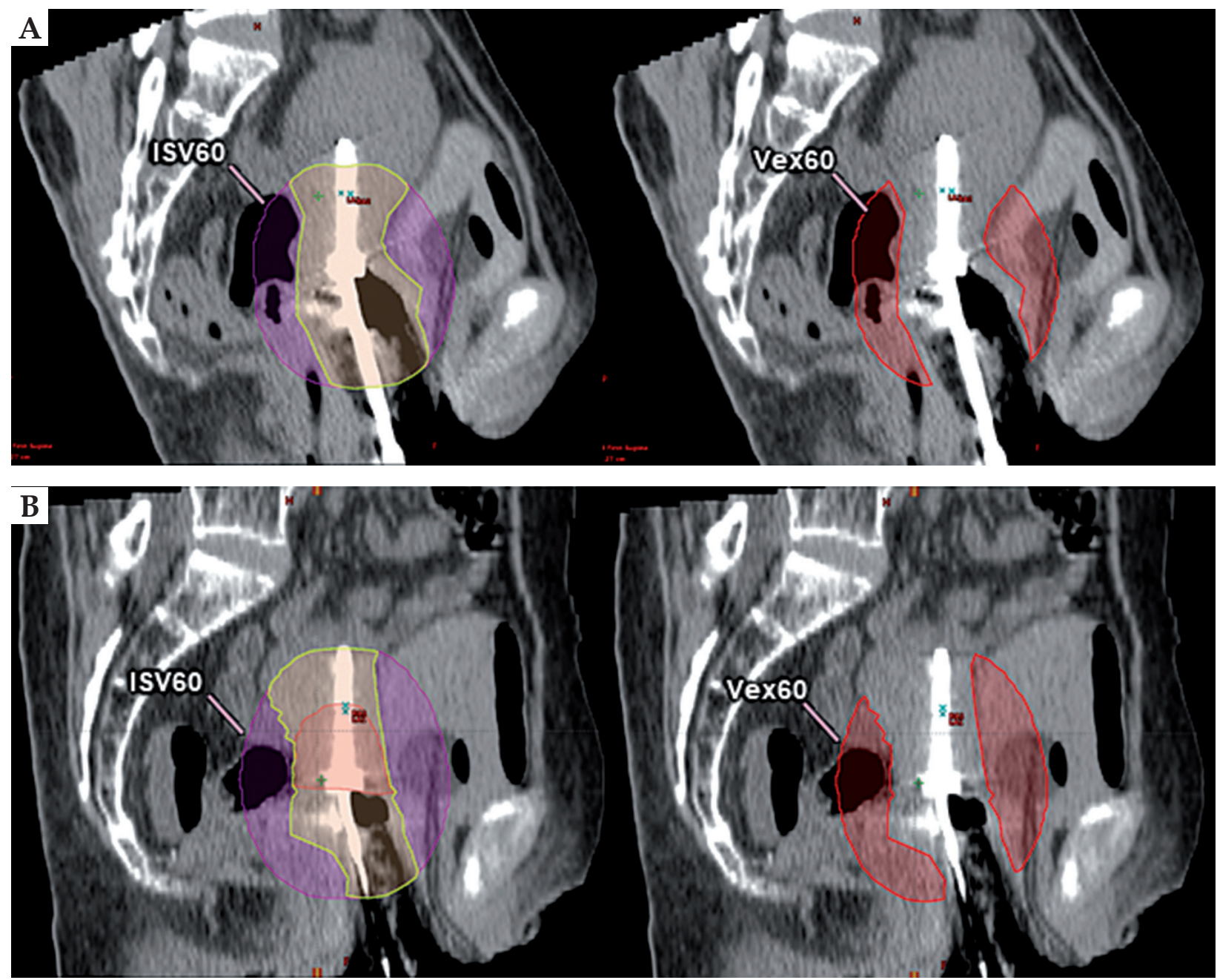

Fig. S1. A, B) Equal ISVs values but different in their conformations between each individual, due to different dose distributions and sizes of individual tumors and pelvic organs; the compositions of Vneg. The varied conformations precipitate the unequal encompassment for organ at risk motion, leading to unequal toxicity correlations. Of note, Rexs and iRexs differed in each case and correlated more to GI toxicity, highlighting the relative property of ratio parameters. As summarize in Table S7

Table S7. Patient characteristic, dose parameters, and radiation toxicity according to cases presented in Fig. S1

\begin{tabular}{|c|c|c|c|c|c|c|c|c|c|c|c|c|}
\hline Patient & $\begin{array}{l}\text { Age } \\
\text { (year) }\end{array}$ & Stage & $\begin{array}{l}\text { Vneg } \\
\text { (cc) }\end{array}$ & $\begin{array}{l}\text { HR-CTV } \\
\text { (cc) }\end{array}$ & $\begin{array}{l}\text { ISV60 } \\
\text { (cc) }\end{array}$ & $\begin{array}{l}\text { Vex60 } \\
\text { (cc) }\end{array}$ & Rex60 & iRex60 & $\begin{array}{c}\mathrm{D}_{2 c c} \\
\text { rectum } \\
(\mathrm{Gy})\end{array}$ & $\begin{array}{c}\text { Gl } \\
\text { toxicity } \\
\text { grade }\end{array}$ & $\begin{array}{c}\text { GU } \\
\text { toxicity } \\
\text { grade }\end{array}$ & $\begin{array}{l}\text { F/U time } \\
\text { (month) }\end{array}$ \\
\hline S1-A & 59 & $3 B$ & 98.31 & 19.5 & 215.5 & 116.4 & 1.186 & 2.194 & 72.82 & 0 & 0 & 40 \\
\hline S1-B & 69 & $3 B$ & 87.33 & 25.5 & 215.5 & 127 & 1.446 & 2.461 & 72.78 & $3^{*}$ & 0 & 42 \\
\hline
\end{tabular}

*Telangiectasia at rectum and anal canal 

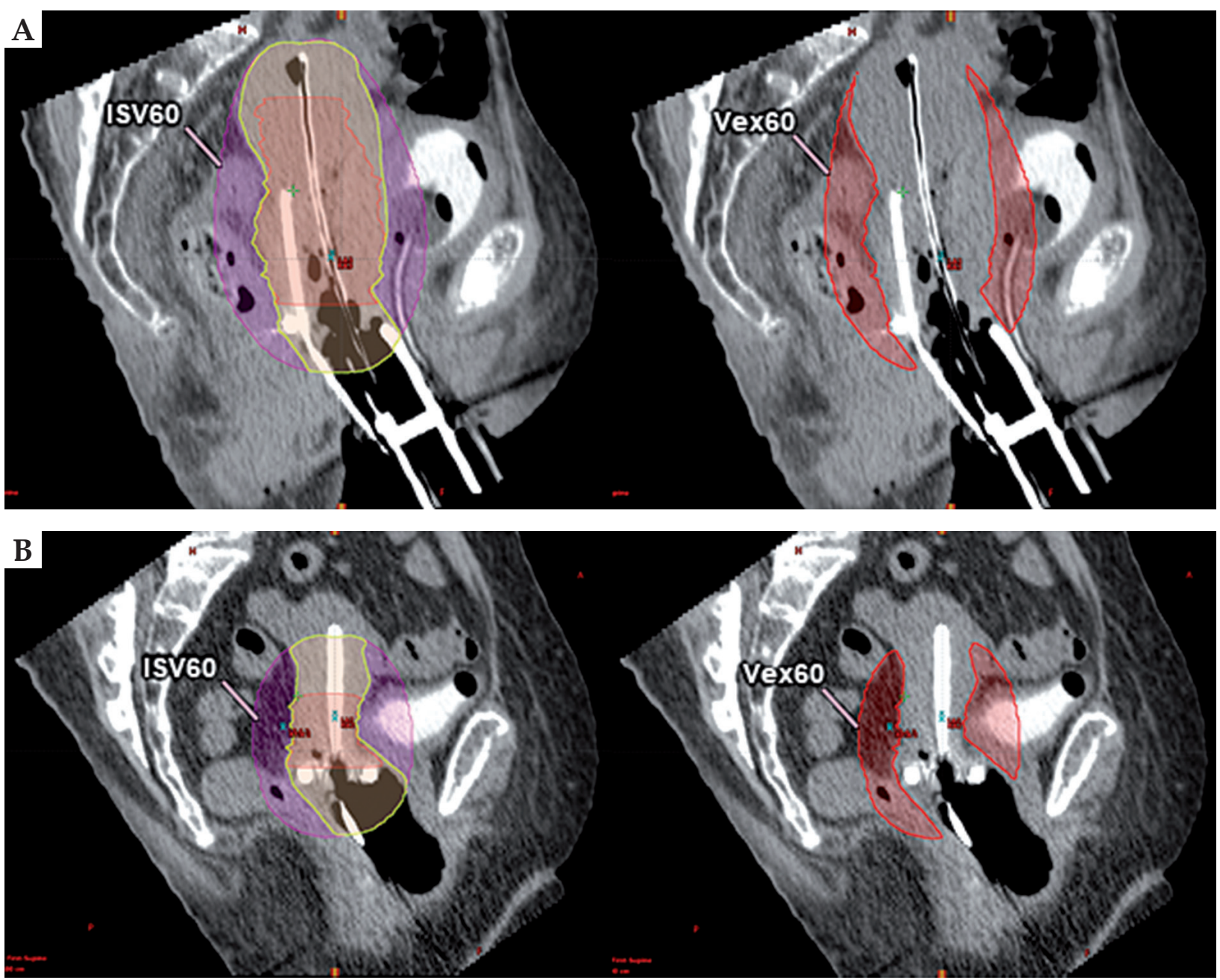

Fig. S2. A, B) Equal Vexs values but different in their conformations between each individual, due to different dose distributions and sizes of individual tumors and pelvic organs; the compositions of Vneg. The varied conformations precipitate the unequal encompassment for organ at risk motion, leading to unequal toxicity correlations. Of note, Rexs and iRexs differed in each case and correlated more to GI toxicity, highlighting the relative property of ratio parameters. As summarize in Table S8

Table S8. Patient characteristic, dose parameters, and radiation toxicity according to cases presented in Fig. S2

\begin{tabular}{lcccccccccccc} 
Patient & $\begin{array}{c}\text { Age } \\
(\text { year })\end{array}$ & Stage & $\begin{array}{c}\text { Vneg } \\
(\mathrm{cc})\end{array}$ & $\begin{array}{c}\text { HR-CTV } \\
(\mathrm{cc})\end{array}$ & $\begin{array}{c}\text { ISV60 } \\
(\mathrm{cc})\end{array}$ & $\begin{array}{c}\text { Vex60 } \\
(\mathrm{cc})\end{array}$ & Rex60 & iRex60 & $\begin{array}{c}\mathrm{D}_{2 c c} \\
\text { rectum } \\
(\mathrm{Gy})\end{array}$ & $\begin{array}{c}\mathrm{Gl} \\
\text { toxicity } \\
\text { grade }\end{array}$ & $\begin{array}{c}\mathrm{GU} \\
\text { toxicity } \\
\text { grade }\end{array}$ & $\begin{array}{c}\mathrm{F} / \mathrm{U} \text { time } \\
\text { (month) }\end{array}$ \\
\hline S2-A & 76 & 3B & 244.3 & 160.5 & 406.25 & 158.71 & 0.649 & 1.663 & 69.73 & 0 & 0 & 41 \\
\hline S2-B & 68 & 3B & 85.77 & 95 & 245.25 & 158.97 & 1.859 & 2.864 & 75.03 & $3^{*}$ & 0 & 34
\end{tabular}

*Telangiectasia at rectum and sigmoid colon 